\title{
COMPORTAMENTO ALIMENTAR E DIETA DE SERPENTES, GÊNEROS BOIRUNA E CLELIA (SERPENTES, COLUBRIDAE) ${ }^{1}$
}

\author{
Carla da Costa Pinto ${ }^{2}$ \\ Thales de Lema ${ }^{3}$
}

\begin{abstract}
FEEDING BEHAVIOR AND DIET OF SNAKES, GENERA BOIRUNA AND CLELIA (SERPENTES, COLUBRIDAE). Boiruna maculata Boulenger, 1896 and Clelia rustica (Cope, 1878) were observed in captivity feeding snakes and rodents, respectively. Both species have shown a similar procedure in relation to the prey. Major behavior differences among the two species were: rodents killed before being swallowed, and snakes were mostly swallowed alive; both species are able to find the rodents head faster than the snake one; the coils formed during constriction were also used to hold the prey, specially the last coil, while swallowing rodents. Informations on stomach contents was gathered by dissection of preserved specimens of Clelia clelia (Daudin, 1803 ) and C. plumbea (Wied, 1820) were also included in the dietary study. The majority of preys consisted on snakes and lizards. Other prey items were mammals and birds. Adult snakes prey relatively smaller animals than the juvenile snakes do.
\end{abstract}

KEYWORDS. Boiruna, Clelia, food, feeding, behavior.

\section{INTRODUÇÃO}

Sobre a dieta alimentar, conteúdo estomacal e comportamento alimentar de serpentes meridionais, há descrições de Amaral (1924), Schouten (1931), Ananjeva \& Orlov (1982), Vitt \& VANGilder (1983), Lema et al. (1983), Henderson et al. (1987), MushinsKy (1987) e SAZIma (1989a, b). Atualmente, as análises populacionais (taxocenose, história natural) vêm contribuindo para o conhecimento da alimentação de serpentes (STRÜSSMANN \& SAZIMA, 1993). A alimentação especializada, como a ofiofagia, foi abordada por GreENE (1983), dificultando a comparação com as de hábitos generalistas (SAVITZKY, 1983). Na Região Neotropical, são conhecidas espécies ofiófagas dos gêneros Boiruna Zaher, 1996, Clelia Fitzinger, 1826, Erythrolamprus Boie, 1826, Micrurus Wagler, 1824 e Phalotris Cope, 1861 (Abalos \& NAder, 1968; Greene, 1983; Lema et al., 1983; Mushinsky, 1987). É grande a diversidade de métodos de captura e subjugação da presa (Ruben, 1977),

1. Parte da Dissertação de Mestrado, Faculdade de Biociências, Pontifícia Universidade Católica do Rio Grande do Sul (PUCRS), Porto Alegre, RS

2. In memoriam.

3. Faculdade de Biociências, PUCRS, Av. Ipiranga 6681, Prédio 40, Lab. 152, C. P. 1429, 90619-900 Porto Alegre, RS, Brasil. (crothales@pucrs.br) 
tendo sido observados dois principais tipos de imobilização, a constrição e o envenenamento (RoDRíGUEZ-ROBLES, 1992). A constrição é o comportamento pelo qual a serpente imobiliza sua presa com pressão exercida por dois ou mais pontos de seu próprio corpo (GREene \& BurghaRdT, 1978), ocorrendo amplamente em espécies de Boidae e Colubridae (Amaral, 1924; Abalos \& NADER, 1968; Herzog \& Burghardt, 1974; Greene \& Burghardt, 1978; Lopes et al., 1991). Esse processo pode ser classificado em três padrões, conforme o lado do corpo da serpente utilizado para formar a espira: ventre, dorso ou voltado na direção da própria cabeça, de forma irregular, sem definir face do corpo utilizada (WILLARD, 1977). Vários autores divergem sobre o modo de constrição e a presença ou não de lateralidade nas diversas espécies de serpentes (WILLARD, 1977; Greene \& Burghardt, 1978; Heinrich \& Klassen, 1985).

Boiruna maculata (Boulenger, 1896) e Clelia spp. utilizam a constrição das presas (WILlARD, 1977) e apresentam hábito alimentar generalista, no qual se incluem serpentes, sáurios e roedores (AMARAL, 1924; VitT \& VANGILDER, 1983; HAlloy \& LAURent, 1984). A alimentação de B. maculata e C. plumbea (Wied, 1820) foi tratada por ABAlos \& Nader, 1968; Vitt \& Vangilder, 1983; Halloy \& Laurent, 1984 e Mushinsky, 1987 e detalhes dos mecanismos de comportamento, por meio dos quais elas subjugam e ingerem suas presas, foram examinados por LEMA et al. (1983).

Analisaram-se o comportamento predatório e a dieta de B. maculata e C. rustica (Cope, 1878), seus métodos de dominação das presas, sua preferência alimentar e a correlação entre as dimensões e a massa da presa e do predador, além do registro dos itens do conteúdo estomacal dessas duas espécies e de C. plumbea e C. clelia (Daudin, 1803).

\section{MATERIAL E MÉTODOS}

Foram observados 27 exemplares jovens e adultos vivos de Boiruna maculata e 12 adultos de Clelia rustica, medindo de 48 a $131 \mathrm{~cm}$ e pesando de 39 a $886 \mathrm{~g}$ e mantidos em cativeiro de um a 36 meses. O material estudado é oriundo das instituições: Laboratório de Venenos da Seção de Herpetologia, Instituto Butantan, São Paulo (IBSP); Biotério do Departamento de Zoologia, Universidade Estadual de Campinas (UNICAMP); Museu de Ciências Naturais, Fundação Zoobotânica do Rio Grande do Sul (MCNZ); Departamento de Zoologia da Universidade Estadual Mesquita Filho, Rio Claro (DZRC). Algumas observações foram realizadas no Instituto Butantan e no Departamento de Zoologia da UNICAMP.

Foram dissecados exemplares das coleções IBSP (29117, 29253, 30312, 34092, 49046); MCNZ (2636, 4093, 6705, 8723); Museu de Ciências e Tecnologia, Pontifícia Universidade Católica do Rio Grande do Sul, Porto Alegre, MCTP (5616); Museu de História Natural de Capão da Imbuia, Curitiba, MHNC (198, 861); Museu Nacional, Universidade Federal do Rio de Janeiro, MNRJ (2382, 2384, 2673 3896, 3934, 3898); Museu de História Natural, UNICAMP, Campinas, ZUEC (1325). Foi examinado o trato gástrico de 210 espécimes sendo 115 de B. maculata; 25 de C. clelia; 42 de C. plumbea e 28 de C. rustica. Para exame do conteúdo estomacal foi feito corte de cerca de $10 \mathrm{~cm}$ na linha mediano ventral no terço médio do tronco. Cada item alimentar foi retirado e determinada a espécie do mesmo, medido e pesado. Os animais vivos utilizados para alimentar as muçuranas foram serpentes da família Colubridae medindo de 14 a $38 \mathrm{~cm}$ e pesando de 6 a $125 \mathrm{~g}$ e camundongos de biotério Mus musculus (Linnaeus, 1758), com 8 a $9 \mathrm{~cm}$ e pesando de 25 a $30 \mathrm{~g}$.

Cada observação foi chamada de sessão alimentar, evitando-se interferir no ambiente experimental, conforme recomendações de MuRPHY \& CAMPBell (1987). Nas sessões em terrário, as presas eram levadas à serpente pela mão do observador, que as prendia até que a muçurana desferisse o bote; nas sessões fora de terrário, usou-se o piso do recinto ou bandeja de plástico com serrapilheira como substrato, onde era colocada e mantida calma, para após ser-lhe oferecida a presa.

Cada sessão foi feita a intervalos médios de 20 dias, oferecendo-se uma presa por sessão, geralmente à tarde, entre 15 e $18 \mathrm{~h}$, de outubro de 1990 a abril de 1993. Numa primeira fase foram oferecidas serpentes e posteriormente roedores. Os critérios utilizados para a seleção de exemplares foram o acesso fácil e manuseio no biotério e animais que comiam regularmente. 
Em sessões de observação visual e direta, conforme método de LeHNER (1979), realizaram-se registros fotográficos, filmagem em vídeo ou desenhos e tomados dados quantitativos como o período necessário para subjugação e ingestão da presa, dados qualitativos como a parte do corpo da presa atingida pela mordida, parte em que foi iniciada a deglutição, transcurso da constrição e qual a face do corpo que foi utilizada para contatar a presa. Anotavam-se a orientação da presa dentro do tubo digestivo, conforme a posição da cabeça da mesma, ou das partes restantes (escamas, penas, pêlos) e graus de digestão dos animais. Estes foram estabelecidos, conforme o estágio da digestão: pouco digerida, praticamente íntegra; semi-digerida, apresentando corpo e cauda e muito digerida, com partes do corpo e/ou da cauda. A identificação das muçuranas foi feita conforme Peters \& Orejas-Miranda (1970), Vanzolini (1986) e Zaher (1996). A determinação das presas foi realizada com base em Peters \& Orejas-Miranda (1970) para ofídios, e em Emmons \& FeER (1990) para roedores.

Medidas de comprimento rostro-anal (CRA), foram aferidas em centímetros e tomadas da tangente ao focinho, até a linha transverso-mediana da escama cloacal. A pesagem foi feita com dinamômetros de $125 \mathrm{~g}$ e $1000 \mathrm{~g}$, com a presa ou predador acondicionado em saco plástico previamente pesado. Para exemplares que excediam $1000 \mathrm{~g}$, usou-se balança de prato. Esse procedimento foi empregado quando a presa estava pouco digerida; caso estivesse muito digerida, usou-se exemplar de dimensão e massa similares como estimativa. Os valores obtidos foram interpretados no estudo da dieta conforme Greene (1983) e Pough \& Groves (1983). Os valores CRA e as massas ( $\mathrm{g}$ ) das presas e dos predadores foram analisados aplicando-se o teste estatístico linear entre duas variáveis (R) conforme Pough \& Groves (1983).

\section{RESULTADOS E DISCUSSÃO}

Comportamento alimentar com presas serpentes (figs.11-16). Na presença da presa em potencial, a muçurana dardeja freqüente e rapidamente a língua, movendo o terço anterior do corpo em várias direções. Após a presa movimentar-se, acerca-se ou fica imóvel. Quando a distância entre ambas fica entre 10 a $20 \mathrm{~cm}$, a serpente desfere o bote, mordendo em qualquer parte da presa. Ao mesmo tempo, ao morder, a muçurana lança o terço anterior de seu corpo circundando a presa e formando duas a quatro espiras, constringindo-lhe o corpo. Na maioria das vezes as espiras são dextrógiras, isto é, o lado direito da muçurana contata com a presa (fig.3). Em todas as observações, o predador formou anéis de constrição com a face dorsal voltada para sua cabeça (fig.1). A constrição da presa pela predadora é mantida até que aquela diminua a resistência, ocasião em que a muçurana busca a cabeça e a abocanha para iniciar a deglutição. A muçurana, dardejando a língua com bastante freqüência, vai se orientando sobre o corpo da presa, movendo seu terço anterior em várias direções e sentidos até que, encontrando a cabeça, abocanha-a (a) (figs. 2, 3); caso não encontre a cabeça, toca em alguma parte do corpo da presa por meio dos escudos rostral, labiais ou gulares, em um ou mais pontos do corpo da presa, como que "tateando" (b). Se ainda não encontra a cabeça da presa, ela a abocanha pelo meio do corpo, soltando e abocanhando novamente mais ao lado, deslocando o local da mordida, repetindo até se aproximar da cabeça (c) (figs. 6, 7), como observado em Micrurus altirostris (Cope, 1860) por Lema et al. (1983). Os dois primeiros procedimentos $(\mathrm{a}, \mathrm{b})$ foram observados para presas como serpentes e roedores, mas o terceiro (c), apenas para serpentes. A dificuldade para a muçurana encontrar a cabeça de presa tipo serpente aumentava quando esta ocultava-a em suas espiras corpóreas, ou sob o corpo da predadora, ou tentando afastar-se com a cabeça esticada, fora do alcance da muçurana. Após ter engolido cerca de um terço da presa, a muçurana afrouxa lentamente as espiras até soltálas completamente (fig. 4). A deglutição conclui-se por ondulações atrás da cabeça da predadora, cuja musculatura, ao distender-se, estira-a para a frente, fazendo com que a presa escorregue inteiramente para dentro do tubo digestivo. Numa sessão foi observada 
B. maculata adulta, de porte muito maior que sua presa, uma Sibynomorphus sp. sem ocorrência de espiras, sendo que a muçurana mordeu a presa próximo à cauda e manteve a boca fechada, apertada, enquanto a presa movia-se em direção oposta, mas sem deslocarse, impossibilitada pela predadora. Após alguns minutos, a presa foi diminuindo os esforços de fuga e a predadora buscou-lhe a cabeça, abocanhando-a e iniciando a ingestão (fig. 5).

Comportamento alimentar com presas roedores. A aproximação, o bote e a formação das espiras para constrição são similares aos detectados para presas serpentes (figs. 6, 7). Após o desfecho do bote, a serpente lança as espiras e depois permanece constringindo até matar o roedor. A seguir, busca a cabeça do roedor, abocanha-o pelo focinho e inicia a ingestão (fig. 3). Com alguma freqüência, o roedor ficava totalmente enovelado pelas espiras, inclusive a cabeça oculta, dificultando seu encontro. Uma vez localizada a posição da cabeça da presa, desfazia parcialmente as espiras e, iniciada a ingestão, a predadora ia afrouxando lenta e paulatinamente as espiras, mas sempre mantendo o último anel apertado, até não ser mais possível prosseguir a ingestão sem afrouxá-lo (figs. 4, 5). Quando a última espira é solta, a serpente pode utilizar uma volta de seu corpo como ponto de apoio para prosseguir a deglutição (figs. 8,9). A conclusão é igual à observada na deglutição de serpentes. Em determinada sessão, o roedor já estava morto e a predadora (C. rustica) soltou as espiras dextrógiras, para logo formá-las antes de iniciar a deglutição e nesse processo utilizou o lado esquerdo de seu corpo mas, não conseguindo iniciar a ingestão, desfez as espiras, refazendo-as pelo seu lado direito. Aparentemente, a difículdade da muçurana fora tentar deglutir a presa pela cauda, pois, ao final a serpente buscou a cabeça e deglutiu-a facilmente.

As muçuranas orientam-se, aparentemente, em direção a sua presa por meio do movimento da mesma. Quanto mais ativa a presa mostrar-se, mais rapidamente é percebida e desferido o bote pela muçurana. Para MCDonald (1973), o movimento da presa não apenas facilita sua localização, como desperta a resposta do bote ("attack response"), ocorrendo com mais rapidez na presa que se movimenta. Ao iniciar a apreensão são formadas duas a quatro espiras, conforme o tamanho da presa, que são utilizadas para subjugação, ou resultam da força aplicada à constrição diante da resistência oferecida pela presa (LOPES et al., 1991). LOOP \& BAILEY (1972) demonstraram que há probabilidade da constrição aumentar com o aumento do tamanho da presa (caso de várias espécies de Boidae e Colubridae). Serpentes não-peçonhentas, ao matarem presas ectotérmicas por constrição, freqüentemente despendem tempo muito prolongado ou alto consumo energético; conseqüentemente, serpentes ofiófagas geralmente ingerem presas vivas ou as matam por esmagamento parcial com suas fortes mandíbulas (MusHINSKY, 1987). As muçuranas, ao ingerirem suas presas ainda vivas, mas debilitadas, eliminam ou reduzem a fase de constrição, diminuindo assim o tempo de manipulação da presa e o custo metabólico de predação (QueIroz, 1984). A constrição é forte o suficiente para matar vertebrados endotérmicos (MusHINSKY, 1987), como observado no caso de presas roedores. QueIroz (1984) relatou que Pituophis melanoleucus (Daudin, 1803) usa mais vigor no apresamento de camundongos adultos do que em jovens de ratos, e que as presas eram mortas antes da ingestão. Os roedores são considerados presas potencialmente perigosas para as serpentes e, ingerindo-os mortos, diminuem os riscos de retaliação e evitam dificuldades na ingestão (ChISZAR et al., 1976; SAZIMA, 1989a). Sobre o uso das espiras e conforme o estabelecido por WiLLARD (1977), as duas espécies aqui analisadas exibiram o padrão (b) relatado. Não há consenso sobre a presença ou não de lateralidade em diversos grupos de 

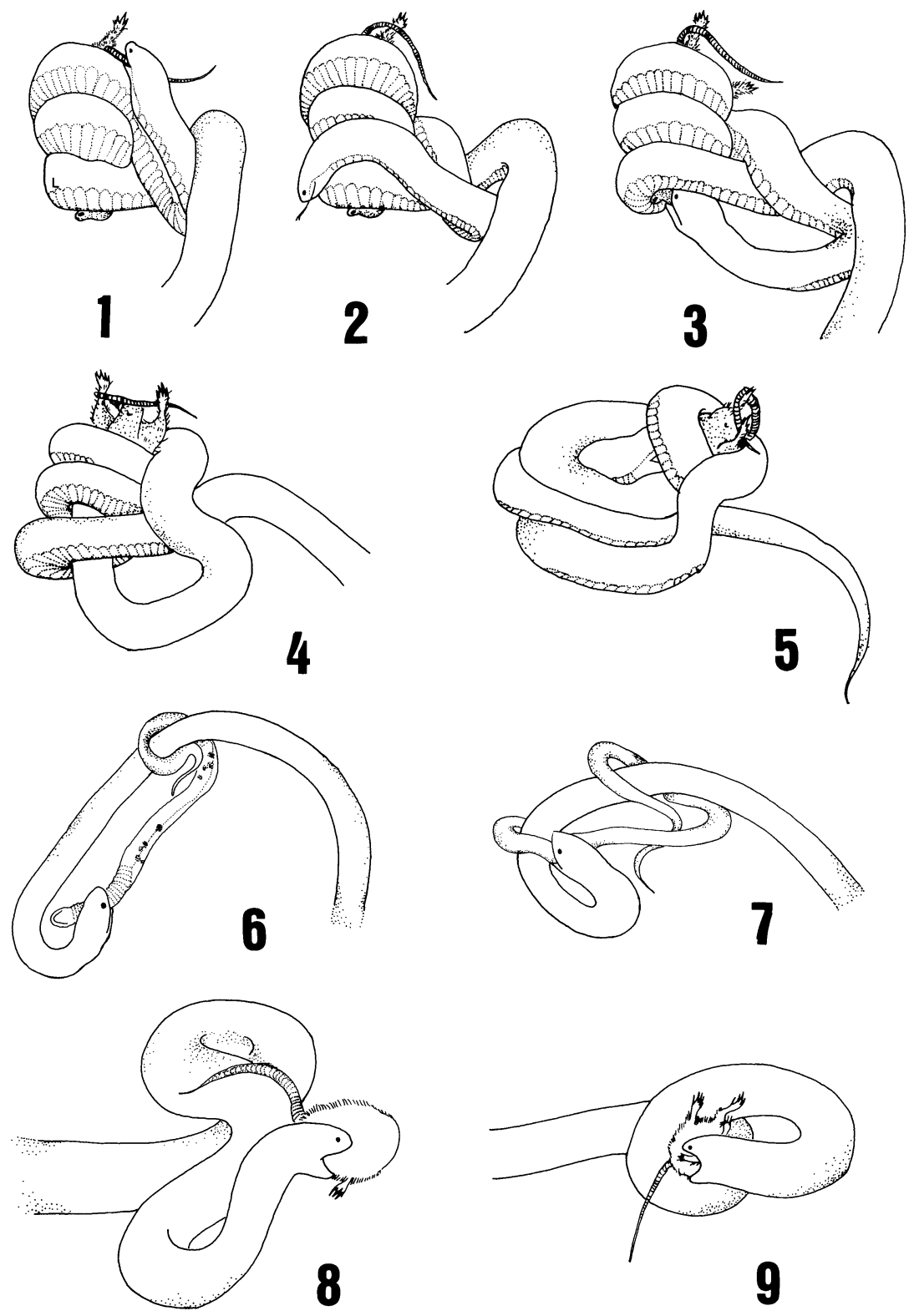

Figs. 1-9. Seqüência comportamental da ingestão de roedor por Clelia rustica: 1, mordida e constrição da presa; 2, busca da cabeça da presa após sua morte, dardejando a língua; 3 , ingestão; 4 , afrouxamento das espiras; 5, fixação com a última espira. Boiruna maculata apresando pequeno colubrídeo sem usar constrição: 6 , deslocamento com mandíbulas em busca da cabeça da presa; 7, início da digestão. Muçurana concluindo ingestão de roedor, auxiliando-se com volta do corpo: 8, desfazendo espiras; 9 , início digestão. 


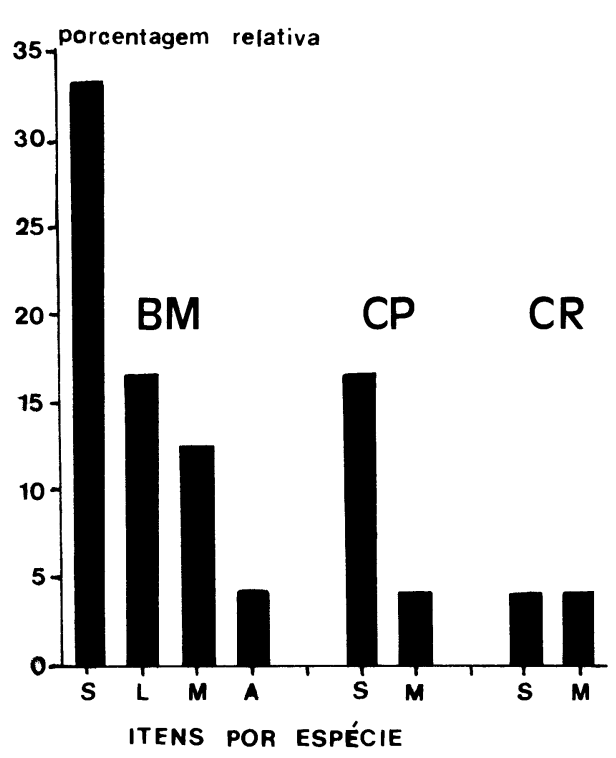

Fig. 10. Tipos de presas encontrados nas dietas e suas porcentagens relativas em cada espécie de serpente, sendo: A, aves; BM, Boiruna maculata; CP, Clelia plumbea; CR, Clelia rustica; L, lagartos; M, mamíferos; S, serpentes. ofídios (Willard, 1977; GREene \& Burghardt, 1978; Heinrich \& Klassen, 1985). Willard (1977) examinou a constrição de presas em 95 espécies das famílias Boidae e Colubridae e não observou um lado dominante entre as espécies, nem entre indivíduos. Lopes et al. (1991) argumentaram que tais resultados basearam-se em poucas observações. Heinrich \& Klassen (1985) observaram o comportamento constritor de dez espécies de ofídios (Boidae, Colubridae), concluindo que há preferência definida por uso de um lado em algumas daquelas espécies. A utilização de uma volta de seu corpo por C. rustica, auxiliou apoiar a presa, para concluir a deglutição. QueIroz (1984) registrou P. melanoleucus pressionar a presa contra o substrato, sugerindo que a serpente utiliza esse processo para segurar a mesma ou posicioná-la para ser ingerida. SAZIMA \& HADDAD (1992) mencionaram esse comportamento para Spilotes pullatus (Linnaeus, 1758). A maioria das serpentes segura a presa e a deglute enquanto esta se debate. Para as serpentes existe um risco real de injúria durante a ingestão e várias características anatômicas e de comportamento parecem fornecer alguma proteção contra a presa (Pough et al., 1993). A constrição pode ser vista como uma inovação etológica fundamental na evolução das serpentes (GREENE \& BURGHARDT, 1978). Considerando-se que presas serpentes podem ser ingeridas vivas, no caso citado a serpente reduziu consideravelmente seus gastos metabólicos ingerindo a pequena presa viva. LEMA et al.(1983) verificaram uma B. maculata atacando um exemplar de Liophis jaegeri (Günther, 1858), muito menor que ela, enrolando-a em suas espiras, mas não conseguindo esmagála, por ser muito fina; aparentemente matou-a com a injeção de veneno, pois mordia e ficava mordendo, como que esperando o efeito do envenenamento. A constrição seria uma tática de subjugação aplicada a presas perigosas para CHISZAR et al. (1976), ou de acordo com seu tamanho, o que ainda está duvidoso para emitir uma afirmativa conclusiva. A ingestão iniciando sempre pela cabeça, tanto em presas serpentes, como roedores parece ser geral (GREENE, 1976), porém não foi ainda estabelecido o que controla esse comportamento. Observou-se que a direção da ingestão de roedores por Elaphe climacophora (Boie, 1826) (Colubridae, constritora) é determinada pela forma do corpo da presa e a posição da mordida inicial e, talvez, pela direção dos pêlos do roedor (Carlos Olegário Dieffenbach, com. pess.). GreENe (1976) afirmou que a localização inicial do 
bote e o diâmetro da presa não são relevantes na direção da ingestão por serpentes ofiófagas. Provavelmente, os melhores motivos para definir o comportamento da ingestão pela cabeça, segundo o mesmo autor, sejam: ingerir presas pela cabeça como sáurios e roedores previne que suas patas impeçam ou dificultem a deglutição, pois ficam "travadas"; no caso de serpentes ofiófagas, as escamas ventrais da presa poderiam oferecer resistência; ingerir pela cabeça previne ou diminui dificuldades em lidar com presas astutas e armadas com dentes, potencialmente perigosas para o predador. É possível que engolir pela cabeça reduza o tempo e o esforço necessários à deglutição, baixando os gastos, dando um ganho energético e minimizando o período de aumento da vulnerabilidade ao predador. As muçuranas segundo MusHinsKY (1987) sempre buscaram a cabeça das presas ao iniciarem a ingestão, sendo que os modos de localização variaram conforme as dificuldades para descobrir a cabeça. Muito do repertório comportamental associado à alimentação nas serpentes é exibido em resposta a informações recebidas do órgão de Jacobson. É provável que o dardejar de língua ocorra muito mais freqüentemente do que alguma outra forma de contato durante o comportamento apetitivo de serpentes e alguns sáurios (CHISZAR et al., 1976). O estímulo direcional provável para localizar a cabeça da presa pode ser as escamas em torno do corpo (GreEne, 1976) para presas como serpentes e, talvez, a direção dos pêlos dos roedores (SAZIMA, 1989a). ChISZAR et al. (1976) afirmaram que não se conhece o grau de participação de outros tecidos com função sensora, nas informações coletadas durante a exploração do ambiente por serpentes. Do mesmo modo não se sabe de que forma informações derivadas de outras modalidades sensoriais interagem com a acuidade vômero nasal ou a maneira pela qual esse órgão influencia essas outras modalidades.

Foi possível observar várias posições tomadas por um jovem de $B$. maculata ingerindo um jovem de Philodryas patagoniensis (Girard, 1897), podendo-se notar que a parte posterior do tronco da presa e mesmo da cauda, apoiaram-se e apertaram o corpo da predadora, mesmo estando quase toda interiorizada nesta (figs. 11-16).

Foram registrados 23 itens alimentares (tab.I), sendo identificadas serpentes da família Colubridae em B. maculata (Atractus sp. jovem e adulto, Liophis almadensis (Wagler, 1824), Lystrophis dorbignyi (Dumèril, Bibron \& Dumèril, 1830), Oxyrhopus petola (Linnaeus, 1758), Sibynomorphus mikanii, Thamnodynastes sp., um jovem de Philodryas patagoniensis (Girard, 1857) e um jovem não identificado), em C. plumbea (Echinanthera cyanopleura (Cope, 1885), Liophis miliaris (Linnaeus, 1758) e um adulto não identificado) e em C. rustica (Oxyrhopus rhombifer Dumèril, Bibron \& Dumèril, 1854). Da família Viperidae foi encontrada Bothrops jararaca (Wied, 1824) em $C$. plumbea. Sáurios da família Scincidae (Mabuya sp.), Teiidae (Ameiva ameiva (Linnaeus, 1758) e Tropiduridae (Tropidurus torquatus (Wied, 1820)) foram encontrados em $B$. maculata. Da ordem Mammalia, família Muriidae, foi constatado um exemplar de Akodon serrensis Thomas, 1902), um jovem e um adulto, não identificados, em B. maculata. Em C. rustica, um Oryzomys nigripes (Olfers, 1818) e da família Didelphidae, um jovem de Metachirus nudicaudatus (E. Geoffroy, 1803) foi encontrado em C. plumbea. Uma espécie de Aves, família Gallidae, Gallus gallus Linnaeus, 1758 jovem foi verificado em $B$. maculata (fig. 10).

Do total de 23 presas encontradas, $72,7 \%(\mathrm{n}=17)$ foram ingeridas pela cabeça, $4,5 \%(n=1)$ pela cauda e $22,7 \%(n=5)$ sem identificação da orientação na deglutição. $O$ item ingerido pela cauda foi um jovem de Atractus sp. com cerca de $1 \%$ do comprimento (CRA) da muçurana. 
Tabela I. Número de exemplares (N) dissecados por espécie e percentuais de conteúdos encontrados. $\mathrm{CG}=$ conteúdo gástrico.

\begin{tabular}{lrrc}
\hline Espécie & $\mathrm{N}$ & $\mathrm{CG}$ & \% de CG \\
\hline Boiruna maculata & 115 & 16 & 13,9 \\
Clelia plumbea & 42 & 5 & 14,2 \\
C. clelia & 25 & 0 & 0 \\
C. rustica & 28 & 2 & 7,1 \\
\hline
\end{tabular}

Tabela II. Razão (R) entre o peso em gramas da presa (P) em relação ao do predador Boiruna maculata (BM), obtida pela divisão $\mathrm{P} / \mathrm{BM}$.

\begin{tabular}{rrccrl}
\hline BM & P & R & BM & P & R \\
\hline 39,0 & 6,0 & 0,15 & 760,0 & 30,0 & 0,03 \\
50,0 & 23,0 & 0,46 & 450,0 & 125,0 & 0,27 \\
112,0 & 14,0 & 0,12 & 450,0 & 125,0 & 0,27 \\
220,0 & 10,0 & 0,04 & 886,0 & 23,9 & 0,02 \\
265,0 & 11,0 & 0,04 & 886,0 & 16,6 & 0,01 \\
620,0 & 43,0 & 0,06 & 886,0 & 1,4 & 0,001 \\
\hline
\end{tabular}

Tabela III. Razão (R) entre o comprimento (CRA em cm) da presa $(\mathrm{P})$ em relação ao predador Boiruna maculata $(\mathrm{BM})$, obtida pela divisão $\mathrm{P} / \mathrm{BM}$.

\begin{tabular}{rccrrr}
\hline BM & P & R & BM & P & R \\
\hline 56,0 & 30,0 & 0,53 & 131,0 & 16,0 & 0,12 \\
69,0 & 24,8 & 0,35 & 48,0 & 8,0 & 0,16 \\
89,0 & 21,8 & 0,24 & 91,0 & 30,0 & 0,32 \\
102,0 & 16,2 & 0,15 & 114,5 & 38,0 & 0,33 \\
112,0 & 16,0 & 0,14 & 114,5 & 32,8 & 0,28 \\
113,6 & 16,2 & 0,14 & 114,5 & 13,5 & 0,11 \\
\hline
\end{tabular}

A relação entre os pesos $(\mathrm{g})$ do predador e da presa apresenta correlação significativa de caráter direto (correlação positiva). Constatou-se que predadores de maior massa ingerem presas de massa também maior (tab. II). Foi registrada uma correlação altamente significativa de caráter inverso (correlação negativa) entre os comprimentos (CRA) da presa e do predador (tab. III). Verifica-se que as muçuranas de maior comprimento alimentam-se de presas relativamente menores que aquelas deglutidas por serpentes de menores dimensões.

A ofiofagia é conhecida nas muçuranas (BRAZIL, 1911; ABALOS \& NADER, 1968; Mushinsky, 1987), assim como a presença de lagartos e roedores em sua dieta (BEEBE, 1946; Halloy \& Laurent, 1984). Estudos sobre a dieta dessas serpentes na região norte (CUNHA \& NASCIMENTO, 1978) e nordeste (VITT \& VANGILDER, 1983) do Brasil, mostraram a mesma diversidade de animais predados por muçuranas. Mais recentemente, TEIXEIRA et al. (1991), registraram ingestão de marsupiais por C. clelia, coletada invadindo ninho desse marsupial. $\mathrm{Na}$ análise aqui apresentada, a dieta de serpentes predominou, apesar de ter sido visto também sáurios, roedores, marsupial e ave. O registro de um jovem de

Gallus gallus em B. maculata deve-se, provavelmente, ao fato da serpente ser tolerada e até estimada nas áreas rurais; o fato foi verificado no interior do Rio Grande do Sul, sugerindo que alguns animais de criadouros domésticos podem ser presas potenciais para as serpentes (LemA et al., 1983).

Dentre o total de conteúdos gástricos $(\mathrm{n}=23), 20,8 \%$ eram presas jovens. Em um dos indivíduos de B. maculata, foram encontrados murídeos adulto e juvenis e, os registros feitos, sugerem que essa espécie é predadora oportunista. A dieta verificada em C. rustica e C. clelia aparenta tratar-se de espécies seletivas.

Nas relações entre as dimensões de presa e predador, constatou-se que as serpentes menores tendem a ingerir presas com peso elevado e comprimento relativamente grande 

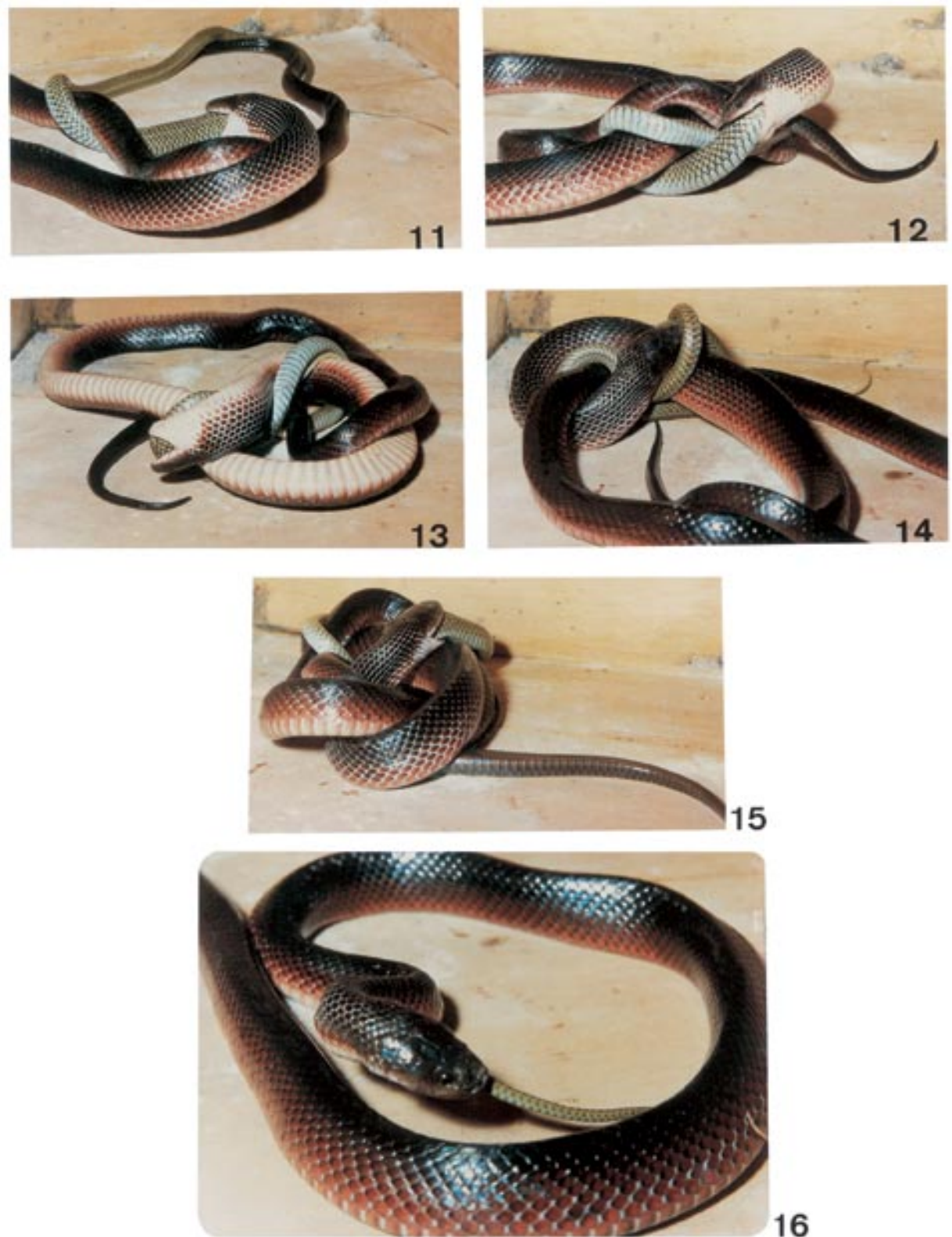

Figs. 11-16. Jovem de Boiruna maculata devorando adulto de Philodryas patagoniensis: 11, dificuldade na constrição pelo menor diâmetro da presa fez afrouxar voltas do corpo enquanto engolia; 12, aperto da muçurana após movimento da presa; 13 , movimentos da presa provocou torcimento da muçurana ficando com o ventre para cima; 14, continuação da constrição; 15 , completo enrolamento da muçurana até a imobilização total da presa; 16, parte final da degluticão e engolimento da presa. 
e que serpentes maiores tendem a apresar animais pesados e de comprimento relativamente pequeno. Dentre os itens que compõem a dieta das muçuranas, os roedores são os de maior comprimento, sendo que os juvenis aparentemente preferem serpentes e essa preferência pode estar relacionada à menor oferta de presas de tamanho apropriado ou ao fato dos roedores serem presas potencialmente perigosas, ocorrendo riscos de retaliação danosas aos jovens, especialmente por serem inexperientes. É possível que presas como serpentes ofereçam menor dificuldade de subjugação e ingestão que os roedores, pois são mais finas e fáceis de enrolar e abocanhar a cabeça. Ao ingerirem presas maiores, as serpentes jovens não necessitam ir em busca de alimento com muita freqüência, o que diminui o risco de exposição aos predadores, embora possam haver problemas durante a ingestão se o tamanho da presa for exagerado em relação à predadora (SAZIMA \& MARTins, 1990). Diversos autores defenderam a idéia que forrageamento e relação presa-predador são as principais pressões ecológicas na evolução das serpentes (MushinsKy, 1987).

Agradecimentos. A Ivan Sazima (Depto. de Zoologia, UNICAMP) pela orientação e uso dos laboratórios. Ao CNPq pelas bolsas de Mestrado e de Pesquisa. A Iara Lucia L. Ferreira, Giuseppe Puorto, Otávio V. Marques e Francisco L. Franco (IBSP) pelo atendimento a consultas. À Moema L. de Araújo (MCNZ), Ulisses Caramaschi (MNRJ), Júlio César de Moura-Leite (MHNC), Lígia Krause (Universidade Federal do Rio Grande do Sul) pelo empréstimo do material. A Miguel T. Rodrigues e Marcelo Duarte (MZUSP) pela identificação do material. A João Luiz Gasparini (UNICAMP) pelos desenhos. À Cristina Arzabe e Denise Freire (UNICAMP) pelo apoio. À Maria Lucia M. Alves (MCNZ) pela revisão do manuscrito. A Júlio César A. González pela determinação de ítens alimentares (Mammalia).

\section{REFERÊNCIAS BIBLIOGRÁFICAS}

Abalos, J. \& Nader, R. 1968. Los habitos de Pseudoboa cloelia y Pseudoboa maculata. Revta Fac. Cienc. med. Univ. nac. Córdoba, Serie Ciencias Naturales, Córdoba, 60:5-12.

Amaral, A. 1924. Contribuição à biologia dos ophidios brasileiros (habitat, hábitos e alimentação). Collnea Trab. Inst. Butantan, São Paulo, 2:177-181.

Ananjeva, N. B. \& Orlov, N. L. 1982. Feeding behaviour of snakes. Vertebr. hung., Budapest, 21:25-31.

BeEbe, W. 1946. Field notes on the snakes of Kartabo, British Guiana, and Caripito, Venezuela. Zoologica, New York, 31:11-52.

Brazil, V. 1911. A Defesa contra o Ophidismo. São Paulo, Pocai \& Weiss. 152p.

CHISZAR, D.; CARTER, T. et al. 1976. Investigatory behavior in the plains garter snake (Thamnophis radix) and several additional species. Anim. Learn. Behav., Stanford, 4 (3):273-278.

Cunha, O. R. \& Nascimento, F. P. 1978. Ofídios da Amazônia. X. As Cobras da Região Leste do Pará. Belém, Museu Par. Emílio Goeldi. 218p. (Publicações Avulsas, 13).

Emmons, L. H. \& Feer, F. 1990. Neotropical Rainforest Mammals: A Field Guide. Chicago, Univ. Chicago. 281p.

GREENE, H. W. 1976. Scale overlap a directional sign stimulus for prey ingestion by ophiophagous snakes. Z. Tierpsychol., Berlin, 41(2): 113-120.

. 1983. Dietary correlates of the origin and raditation of snakes. American Zool., New York, 232(2):431-442.

GreENe, H. W. \& Burghardt, G. M. 1978. Behavior and phylogeny: Constriction in ancient and modern snakes. Science, Knoxville, 200:74-77.

Halloy, M. \& Laurent, R. 1984. Clelia rustica. Revue fr. aquar. Herpetologie, Paris, 11(1):277.

Heinrich, M. L. \& Klassen, H. E. 1985. Side dominance in constricting snakes. J. Herpetology, Chicago, 19(4):531-533.

Henderson, R.W.; Schwartz, A. \& Noeske-Hallin, T. A. 1987. Food habits of three colubrid tree snakes (genus Uromacer) on Hispaniola. Herpetologica, Johnson, 43(2):241-248.

Herzog, H. A., JR. \& Burghardt, G. 1974. Prey movement and predatory behavior of juvenile western yellowbellied racers, Coluber constrictor mormon. Herpetologica, Chicago, 30:285-289. 
Lehner, P. N. 1979. Handbook of Ethological Methods. New York, Garland STPM. 403p.

Lema, T. DE; Araujo, M. L. DE \& Azevedo, A. C. P. DE. 1983. Contribuição ao conhecimento da alimentação e do modo alimentar de serpentes do Brasil. Comun. Mus. Ciênc. PUCRS, Sér. Zool., Porto Alegre, 26:41-121.

LOOP, M. S. \& BAILEY, L. G. 1972. The effect of relative prey size on the ingestion behavior of rodent-eating snakes. Psychon. Sci., Pittsburgh, 28(3):167-169.

Lopes, H. R.; Rocha, C. F. D. \& Abe, A. S. 1991. Constriction behavior in snakes: Is there a side dominance? Revta bras. Biol., Rio de Janeiro, 51(4):853-856.

McDonald, L. 1973. Attack latency of Constrictor constrictor as a function of prey activity. Herpetologica, Chicago, 29:45-48.

Murphy, J. B. \& Campbell, J. A. 1987. Captive maintenance. In: Seigel, R. A.; Collins, J. T. \& Novak, S. S. eds. Snakes: Ecology and Evolutionary Biology. New York, McMillan. p.165-183.

Mushinsky, H. R. 1987. Foraging ecology. In: Seigel, R. A.; Collins, J. T. \& Novak, S. S. eds. Snakes: Ecology and Evolutionary Biology. New York, McMillan. p.302-334.

Peters, J. A. \& Orejas-Miranda, B. 1970. Catalogue of the neotropical Squamata. Part 1, Snakes. Bull. U. S. natn. Mus., Washington, (297):1-347.

Pough, F. H. \& Groves, J. D. 1983. Specialization of the body form and food habits of snakes. Am. Zoologist, New York, 23(2):443-454.

Pough, F. H.; Heiser, J. B. \& McFarland, W. N. 1993. A Vida dos Vertebrados. São Paulo, Atheneu. 839p.

QueIroz, A. 1984. Effects of prey type on the prey-handling behavior of the Bullsnake, Pituophis melanoleucus. J. Herpetology, Chicago, 18(3):333-336.

Rodríguez-Robles, J. A. 1992. Notes on the feeding behavior of the Puerto Rican racer, Alsophis portoricensis (Serpentes: Colubridae). J. Herpetology, Provo, 26(1):100-102.

RuBEN, J. A. 1977. Morpohological correlates of predatory modes in the coachwhip (Masticophis flagellum) and rosy boa (Lichanura roseofusca). Herpetologica, Michigan, 33(1):1-6.

SAvitzky, A. 1983. Coadapted character complexes among snakes; fossoriality, piscivory and durophagy. Am. Zoologist, New York, 23:397-409.

SAzima, I. 1989a. Comportamento alimentar de jararaca, Bothrops jararaca: Encontros provocados na natureza. Ciênc. Cult., São Paulo, 41(20):500-505.

. 1989b. Feeding behavior of the snail-eating snake, Dipsas indica. J. Herpetology, Chicago, 23(4):464468.

Sazima, I. \& Haddad, C. F. P. 1992. Répteis da Serra do Japi: Notas sobre história natural. In: Morellato, L. P. C. ed. História Natural da Serra do Japi. Campinas, Univ. Est. Campinas. p. 212-236.

SAZima, I. \& Martins, M. 1990. Presas grandes e serpentes jovens. Quando os olhos são maiores que a boca. Mems Inst. Butantan, São Paulo, 52(3):73-79.

Schouten, G. B. 1931. Contribuciones al conocimiento de la fauna herpetologica del Paraguay y de los paises limitrofes. Revta Soc. cient. Parag., Asunción, 31(1):5-32.

Strüssmann, C. \& Sazima, I. 1993. The snake assemblage of the Pantanal at Poconé, western Brazil: Faunal composition and ecological summary. St. Neotr. Fauna Environment, Lisse, 28:157-168.

Teixeira, D. M.; Lorini, M. L. \& Porto, M. 1991. Clelia clelia (Muçurana), feeding behavior. H. Review, Oxford, 22(4):131.

Vanzolini, P. E. 1986. Addenda and Corrigenda to the Catalogue of the Neotropical Squamata. Washington, Smiths. Inst. 25 p. [Smithsonian Herpetological Information Service 70]

VitT, L. J. \& VANGiLder, L. D. 1983. Ecology of a snake community in northeastern Brazil. AmphibiaReptilia, Leiden, 4:273-296.

Willard, D. E. 1977. Constricting methods of snakes. Copeia, New York, 1977:379-382.

ZAHER, H. A. 1996. A new genus and species of Pseudoboine snake, with a revision of the genus Clelia (Serpentes, Xenodontinae). Boll. Mus. Reg. Sci. Nat. Torino, Torino, 14(2):289-337. 